\title{
Tratamentos antineoplásicos: foco na perspectiva holística da fé como agente terapêutico
}

\author{
Antineoplastic treatments: focus on the holistic faith perspective as a therapeutic agent
}

\author{
Daniele Andreia Alvares" \\ Graduada em nutrição, Universidade Nove de Julho, São Paulo, São Paulo, Brasil. Autor para correspondência. E-mail: \\ daniele_andreia@hotmail.com
}

\begin{abstract}
Resumo: Introdução: O câncer pode ser considerado como uma massa atípica com crescimento desenfreado com propriedades de autoestimulação, e, Somente a partir da década de 30, durante o Governo Provisório, iniciou-se realmente a batalha contra a carcinogênese, marcada pela construção de hospitais para o tratamento e estudo do câncer. Durante a Idade Média a investigação científica contribuiu para o início do Renascimento, a partir daí a medicina e a fé passaram a ser mais bem compreendidas e incluídas, simultaneamente, no cuidado ao paciente. Objetivou-se, então, através de embasamento na literatura científica, conhecer a relevância e aplicabilidade da fé, concomitantemente as terapias antineoplásicas convencionais. Revisão e discussão: Realizou-se uma revisão bibliográfica em diversas bases de dados, que revelaram que a busca pela fé pode ter nascido da necessidade humana, devido a sua fragilidade, aceitando, mas não cedendo a doença, apresentando os inúmeros benefícios da fé frente a aceitação, tratamento e desfecho positivo, para os pacientes com câncer. Considerações finais: Concluiu-se então que a fé transcende o alívio espiritual e psicológico, modifica o estado físico, atuando nos sistemas: endócrino, imunológico e nervoso central, ajudando na aceitação do diagnóstico e desfechos negativos; aderência e efetividade do tratamento, e o seu resultado, assim como o efeito placebo, se dá pelo método de cura mentecorpo, onde, através das crenças e esperanças a nível psicológico, instalam-se diversos efeitos fisiológicos, resultando em melhor qualidade de vida e sobrevida, melhor prognóstico e eficácia terapêutica
\end{abstract}

Palavras-chave: dor do câncer, cura pela fé, terapia combinada, cooperação do paciente, protocolos antineoplásicos, efeito placebo.

\begin{abstract}
Introduction: Cancer can be considered as an atypical mass with unbridled growth with selfstimulation properties, and, only from the 30's, during the Provisional Government, did the battle really begin? Against carcinogenesis, marked by the construction of hospitals for the treatment and study of cancer. During the Middle Ages scientific research contributed to the beginning of the Renaissance, since then medicine and faith were better understood and included, simultaneously, in patient care. The objective was, then, based on the scientific literature, to know the relevance and applicability of faith, concomitantly with conventional antineoplastic therapies. Review and discussion: A literature review was conducted in several databases, which revealed that the search for faith may have been born of human need, due to its fragility, accepting but not giving in to the disease, presenting the numerous benefits of faith in the face of acceptance, treatment and positive outcome for cancer patients. Final considerations: It was concluded that faith transcends spiritual and psychological relief, modifies the physical state, acting in the systems: endocrine, immune and central nervous, helping in the acceptance of the diagnosis and negative outcomes; adherence and effectiveness of the treatment, and its result, as well as the placebo effect, is given by the mind-body healing method, where, through the beliefs and hopes at the psychological level, various physiological effects set in, resulting in better quality of life and survival, better prognosis and therapeutic efficacy.
\end{abstract}

Keywords: cancer pain, heal by faith, combination therapy patient's cooperation, antineoplastic protocols, placebo effect.

\section{Introdução}

\section{Fisiopatogenia do câncer}

Segundo Willis (1952), o câncer pode ser considerado como uma massa atípica que tem um crescimento desenfreado com propriedades de autoestimulação. 
Essa massa divide-se extraordinariamente, devido a falhas no ciclo celular, perdurando além dos seus limites normais, consequência das anormalidades das vias apoptóticas, além de manifestar instabilidade genética, baseando-se em alterações cromossômicas ou genéticas e transcricionais, desencadeando o surgimento de proteínas anômalas e alterando a produção de outras proteínas importantes para a homeostase celular (Hoff, 2013).

Quanto ao risco genético, é comprovado cientificamente que o surgimento da oncogênese, é o resultado da interação entre está e os fatores ambientais aos quais o indivíduo é exposto durante sua vida.

Pode-se declarar que a carcinogênese tem característica fenotípica e genotípica, herdáveis ou não (Oliveira Santos, 2018).

Os hábitos e culturas alimentares implantados desde cedo na rotina de um indivíduo, podem impactar no surgimento do câncer, seja pelo tempo de exposição a substâncias carcinogênicas, baixo consumo de alimentos preventivos, ou, pelo desbalanço energético e respostas metabólicas e hormonais (Oliveira Santos, 2018).

Os nutrientes e a quantidade dos alimentos, seu modo de preparo, o balanceamento calórico, sua conservação, entre outros aspectos, são fatores relevantes na carcinogênese (Oliveira Santos, 2018).

Quanto ao diagnóstico, a descoberta precoce de lesões em fases iniciais é fundamental, por isso, os profissionais da saúde devem ser capazes de reconhecer alguns sinais e sintomas, tais como: febre continua, nódulos, feridas com difícil cicatrização, dispepsia recorrente e disfonia, previamente aos que indiquem lesões em estágios mais graves, como o sangramento, obstrução intestinal, respiratória e dor (Oliveira Santos, 2018).

As neoplasias podem ser benignas ou malignas. Os benignos se caracterizam por terem crescimento lento e dilatável, normalmente calcando o órgão adjacente, possuindo limites bem definidos. Porém, o maligno, tem um crescimento célere, invadindo órgãos adjacentes e vasos linfáticos, sendo temido por sua capacidade metastática. Além da sua capacidade intrínseca e extrínseca (Hoff, 2013).

Intrinsecamente, as células são capazes de se autorregeneração indefinida, crescimento autossuficiente, alta tolerância a fatores antiproliferativos, indiferença a apoptose e ao sistema imune, alterações metabólicas adaptativas e variações genéticas. Extrinsecamente, possuem a habilidade de ativar a angiogênese, indiferença ao ataque do sistema imunológico contra tumores, ajuste da resposta inflamatória e do reparo tecidual, agregação celular durante a invasão e metástase (Hoff, 2013).

\section{História e quadro atual do câncer}

Del Volgo (1998), enfatizava que para os egípcios, a saúde estava relacionada ao “bem” e a doença ao “mal”. Para os Greco-romanos, a doença era o resultado da quebra do equilíbrio universal e a saúde a plena felicidade.

Somente a partir da década de 30, durante o Governo Provisório, iniciou-se realmente a batalha contra a carcinogênese, marcada pela construção de hospitais para o tratamento e estudo do câncer (Oliveira Santos, 2018).

O conceito de saúde, como sendo algo muito mais complexo que somente a inexistência da doença, consolidou-se, a partir de 1946, originando-se as discussões acerca da criação de clínicas especializadas. Contudo, devido os conceitos da medicina liberal, a partir de 1960, o assunto foi tratado como de natureza especifica (Oliveira Santos, 2018).

Em 2001, um estudo realizado em Harvard, estipulou que alguns fatores de risco, como dieta, inatividade física, álcool, tabaco, doenças sexualmente transmissíveis, condições ambientais e doenças infecciosas (estima-se que cerca de $18 \%$ dos casos de câncer, a nível mundial, têm origem em agentes infecciosos), seriam responsáveis por cerca de 35\% da mortalidade mundial por neoplasias (Oliveira Santos, 2018).

\section{A medicina e a fé}

Durante a Idade Média, em tudo o homem via a intervenção divina ou do demônio, tudo era dividido entre bem o mal, seu conhecimento era empírico e baseava-se na crença do sobrenatural e vida eterna. Porém, alguns acreditavam que conhecer profundamente a natureza e o que a cercava, permitia que ascendessem mais rapidamente para perto do Criador. Estes grupos de investigação científica contribuíram para o início do Renascimento (Fernandes, 2000).

Atualmente diversos estudos têm validado que pacientes que possuem algum tipo de fé, mesmo que seja na cura obtida pelos homens e pela medicina, independentemente da predileção religiosa, possuem maiores chances de sobrevida do que os materialistas. A compreensão dessa necessidade por parte do paciente, que ultrapassa as exigências físicas, que atinge um patamar espiritual, se torna crucial para potencializar os protocolos de tratamento, levando humanização para a relação médico-paciente (Braile, 2013). 
Farris (1996) observou que as pessoas precisam passar por momentos difíceis para alcançarem a sua plenitude emocional e espiritual, onde, cada um tem a opção de ultrapassá-la positiva ou negativamente, destacando ainda, que os detentores de algum tipo de fé possuíam maiores habilidades para sobrepujar as dificuldades e obterem melhor prognóstico.

A espiritualidade é a associação dos valores emocionais, morais e mentais, que permeiam o comportamento, pensamentos e atitudes diante das adversidades. Inúmeros estudos científicos comprovaram que o poder do perdão, da superação, esperança e resiliência agem sobre a homeostase do sistema nervoso e endócrino, diminuindo a liberação de adrenalina e cortisol, levando a um desfecho mais favorável e com melhor prognóstico (Xavier, 2009).

Desta forma, o objetivo do trabalho foi demonstrar, através de embasamento na literatura científica, a relevância e aplicabilidade da firme convicção, como coadjuvante das terapias antineoplásicas protocoladas e padronizadas (cirurgia, quimioterapia etc.).

\section{Revisão}

Fez-se uso do método Revisão Bibliográfica, utilizando-se de periódicos e fontes impressas, como livros e revistas.

A pesquisa foi orientada pelos seguintes descritores: dor do câncer, cura pela fé, terapia combinada, cooperação do paciente, protocolos antineoplásicos e efeito placebo.

Do total de trabalhos que continham tais descritores, selecionou-se os 66 estudos presentes, quanto aos impressos, optou-se por selecionar os livros que continham trechos que fossem relevantes para enriquecer e aclarar as ideias advindas das outras fontes de pesquisa, servindo de embasamento no presente artigo.

Tal tipo de pesquisa é descrito na literatura como consentâneo, pelo fato do grande alicerce expansivo que tal estilo possibilita ao pesquisador. Além da praticidade, no que tange a desnecessidade de deslocamento para coleta de dados.

Destaca-se ainda que a revisão bibliográfica é de extrema importância - pois qualquer pesquisa num dado momento pode ser qualificada embasamento científico - uma vez que a estrutura para quaisquer linhas de pesquisa, é uma busca criteriosa e fidedigna de dados feito sobre o tema escolhido.

\section{Discussão}

\section{Tratamentos antineoplásicos}

Os tratamentos mais citados na literatura são: a cirurgia, a radioterapia, a quimioterapia e a hormonioterapia, contando também com uma equipe transdisciplinar, incluindo o nutricionista. Sendo essa interdisciplinaridade mais factível do que uma assistência isolada no cuidado com o paciente (Oliveira Santos, 2018). Ainda nessa questão, nos últimos anos tem-se discutido, dentro e fora da medicina, sobre o efeito placebo e a fé como possíveis adjuvantes no tratamento neoplásico, temática que será abordada nos próximos tópicos.

Um estudo realizado por Bernardes (2017), aponta que os tratamentos em conjunto mais utilizados pela medicina são: quimioterapia e radioterapia $(22,8 \%)$ e a cirurgia adjunta a quimioterapia $(22,8 \%)$.

Contudo, é de suma importância que a população seja atendida de forma universal e integral, visando o controle da doença, mas, também objetivando proporcionar condições para o bem-estar global do indivíduo portador de câncer e sua família (Oliveira Santos, 2018).

Diante desta realidade torna-se crucial o papel do diagnóstico precoce, para que as chances de sobrevida dos portadores de neoplasias continuem a crescer, pois a identificação do câncer em estágios iniciais possibilita uma maior chance de cura. Um dos problemas mais relevantes ocorre: entre o diagnóstico da doença na atenção primária e a demora para o tratamento com os médicos da área; encaminhamento aos hospitais referência para a realização de cirurgia e outros tratamentos, tornando-se o ponto frágil na estruturação dos acessos aos serviços de saúde (Xavier \& Gentilli, 2012).

Com a finalidade de assistência ao paciente oncológico, foi elaborado a portaria 874/GM, objetivando reduzir a mortalidade e incapacidade causadas pela doença, diminuir a incidência de alguns de câncer e contribuir para um melhor prognóstico aos indivíduos, desde o diagnóstico até a terapia (gratuito, desde as prescrições ao acesso a analgésicos opiáceos ou correlatos) e cuidados paliativos (Junior, Santana, \& Sonobe, 2020). 
Devido a extensão geográfica, desigualdade no acesso aos recursos assistenciais e a alta demanda de atendimento, a qualidade e o tempo de recuperação dos pacientes ficam comprometidos (Coy, 2013).

\section{Terapias Convencionais}

\section{Cirurgia}

Atualmente essa intervenção serve tanto para identificação, quanto tratamento de tumores operáveis. Quanto ao tratamento, pode ser curativo, onde o objetivo primário é a ressecção total do tumor, ou, pode ser paliativo, fazendo descompressão de estruturas vitais, diminuindo a dor e até retirada de uma lesão que causa prejuízos funcionais ao paciente (Novaes et al., 2008).

É necessário, portanto, conhecer previamente a cirurgia e o estadiamento da doença. Para a realização do procedimento é obrigatório a presença de profissionais capacitados, ambiente e instrumentação cirúrgica esterilizados e cuidado no manejo dos tecidos infiltrados (Novaes et al., 2008).

\section{Radioterapia}

A radioterapia baseia-se no uso de radiações ionizantes, usados para extinguir ou obstruir as células neoplásicas. Tal procedimento é indolor e invisível, subdivide-se em: Teleterapia (a radiação é lançada através de um aparelho, focalizado no local específico e com o paciente deitado); e Braquiterapia (é aplicado diretamente no local pelos aplicadores, é feito em nível ambulatorial e pode ser necessário uso de anestesia) (Sawada, Dias, \& Zago, 2006).

Pode ser curativo e Paliativo (visando proporcionar qualidade de vida, exercendo efeito antiálgico, descompressivo e hemostático) (Barboza \& Oliveira, 2006).

Antes da prescrição das radioterapias, alguns aspectos devem ser analisados. Curativo: saber com exatidão a extensão da doença; utilizá-la de forma adjuvante a fim de reduzir as complicações e devido aos danos celulares temporários é necessário estudar qual a melhor frequência de tratamento (Barboza \& Oliveira, 2006).

Paliativo: pode aliviar desconfortos relativos à doença, alcançando até $85 \%$ de eficiência; nas compressões ou obstruções causadas pela neoplasia a radioterapia pode diminuir o tamanho tumoral (Barboza \& Oliveira, 2006).

Seus principais efeitos colaterais são cefaleia, náusea, tontura, disfagia, odinofagia, xerostomia, rouquidão, tosse seca, fraqueza, diarreia, cólica e ardor ao urinar (Willis, 1952).

\section{Quimioterapia}

Terapia baseada no uso de medicamentos que agem de forma generalizada. Esse procedimento tem 3 objetivos principais: Curativo (objetiva extirpar o tumor, mesmo não havendo, em todos os casos, a certeza de cura); Controle da doença (usada para atenuar ou evitar que a neoplasia cresça ou invada outros tecidos) e o Paliativo (usada para aliviar os sintomas causados pelo tumor, em casos mais avançados, contribuir para uma melhor sobrevida do paciente) (Junior et al., 2007).

O oncologista é o profissional habilitado para indicar o melhor medicamento e tratamento (Schein et al., 2006).

Quanto ao medicamento, alguns fatores devem ser levados em consideração na hora da escolha: tipo e estágio do câncer; condição física; outras comorbidades e se o tratamento já foi realizado anteriormente (Junior et al., 2007).

A quantidade é baseada no peso do paciente. Em alguns casos específicos, como idosos, desnutridos, obesos, entre outros, as doses são ajustadas (Junior et al., 2007).

Os ciclos são estabelecidos respeitando o estágio do tumor, acompanhando os efeitos através de exames bioquímicos e clínicos feitos regularmente. Para cada ciclo é necessária uma pausa para que o organismo volte a homeostase (Junior et al., 2007).

Sempre que possível é importante que os ciclos sejam respeitados e completos, porque isso determinará o sucesso do tratamento. Porém, em alguns casos o ciclo tem que ser alterado devido aos possíveis efeitos colaterais que o tratamento causa, além de prescrição de outros medicamentos para atenuar tais complicações (Schein et al., 2006).

Alguns resultados indesejáveis são: dispepsia, disgeusia, alopecia, fadiga, ansiedade, perda de peso, alteração do humor e do sono, anorexia, depressão, constipação, disfagia, diarreia, dispneia, astenia, mucosites e tonturas (Silva et al., 2010). 


\section{Imunoterapia}

Diferentemente dos outros tratamentos, que visam atacar as células tumorais, a imunoterapia assiste ao sistema imunológico do paciente a localizar e erradicar o câncer. O propósito do tratamento é diminuir o tumor e posteriormente tentar erradicá-lo(Veronesi, 1976).

Readaptar o sistema imune requer esforço e tentativas, não sendo indicado em todos os casos, sua indicação depende do tipo do câncer, estádio, e tratamento aplicado no momento (Veronesi, 1976).

A finalidade da imunoterapia é potencializar a defesa contra agentes estressores. Através do bloqueio dos fatores que confundem o sistema imunológico, as medicações potencializam a resposta imune, impulsionando a atuação dos linfócitos e capacitando-os para enxergarem o tumor como um corpo estranho (Veronesi, 1976).

Segundo a revista The Economist, o tratamento pode aumentar, consideravelmente, a expectativa de vida em alguns tipos de câncer. Segundo o estudo, a média de sobrevida, com tratamentos convencionais, era de menos de 5 anos (Carvalho, 2019).

A duração do tratamento depende da ação das drogas e responsividade do paciente, cogita-se que, nos próximos anos, seu uso ultrapasse as outras linhas de tratamento (Carvalho, 2019). Êmese, fadiga e irritação colônica, são os sintomas mais relatados (Freire, 2019).

\section{Hormonioterapia}

Objetiva reduzir ou bloquear a ação de alguns hormônios que, por uma alteração na homeostase, incitam a mitose de células neoplásicas. Alguns hormônios podem ter um papel desencadeador e perpetuador de diversos tumores, tais células cancerosas possuem receptores que atraem os hormônios circulantes, utilizando-os para impulsionar o desenvolvimento celular. A ação da terapia acontece de duas formas: impedir a circulação hormonal, e por conseguinte, sua utilização, ou impedindo que os mesmos se liguem nas células, o tempo de multiplicação celular é reduzido e a apoptose celular é acelerada, levando a regressão do tumor (Leal, Cubero \& Giglio, 2010).

Pode ser usado concomitantemente com os outros protocolos terapêuticos, atenuando o volume total tumoral, preservando a maior parte dos tecidos saudáveis ou após a cirurgia, diminuindo a reincidência (Leal, Cubero \& Giglio, 2010).

A indicação da hormonioterapia depende de alguns fatores. Os comprimidos são administrados diariamente e os injetáveis mensal ou trimestralmente. A interrupção hormonal também pode ser obtida através de cirurgia ou radiação. O tempo de tratamento depende do tipo de doença e é analisado individualmente (Gabriel et al., 2017).

Assim como a imunoterapia, seus efeitos colaterais são menos agressivos: fadiga, alterações menstruais, sudorese excessiva, impotência e alteração do humor (Gabriel et al., 2017).

Mas com a devida avaliação médica, uso de medicamentos atenuadores, manutenção da rotina e prática regular de atividade física, o risco de complicações reduz significativamente, potencializando a eficácia do tratamento (Gabriel et al., 2017).

\section{Dietoterapia}

Um suporte proteico adequado é extremamente importante, pois sua depleção pode levar a desgaste e fadiga muscular o que impacta na eficácia dos antineoplásicos. A recomendação hídrica é parecida com a de indivíduos saudáveis: $1 \mathrm{ml} / \mathrm{Kcal}$ ou $35 \mathrm{ml} / \mathrm{Kg} / \mathrm{P}$, podendo haver ajustes em caso de drenagens, retenções hídricas e grandes perdas (Waitzberg et al., 2004).

A Terapia Nutricional, complemento da dietoterapia, é recomendada para pacientes em tratamento antineoplásico com ingesta oral abaixo do preconizado, ou seja, consumo de menos de $70 \%$ do gasto energético estimado por mais de 10 dias (Waitzberg et al., 2004).

Deve ser iniciada até as primeiras 24 horas, desde que o quadro atual se apresente estável. Prescrevendose uma dieta individualizada, de acordo com o diagnóstico e histórico pessoal (Sociedade Brasileira de Nutrição Parenteral e Enteral, 2011).

São diversos os motivos que levam os pacientes com câncer a desenvolver esse sintoma: náuseas, êmese, xerostomia, odinofagia, alterações metabólicas e efeitos colaterais dos tratamentos antineoplásicos, dor, uso de sedativos e fadiga excessiva (Soratto et al., 2016). Podem ser prescritos antieméticos e agentes orexígenos 
(canabinoides, agentes progestacioanais e corticosteroides), que visam aumentar o apetite (Sociedade Brasileira de Nutrição Parenteral e Enteral, 2011).

Quanto a perda de peso involuntária, muito comum em pacientes oncológicos, pode ser resultado de: alterações metabólicas e hormonais causadas pela doença, como a geração de citocinas pró-inflamatórias (do tumor e do hospedeiro), como TNF- $\alpha$ e IL; hipermetabolismo; tumores sólidos; doença em estadiamento III e IV; presença de metástases; mucosites; saciedade precoce; náuseas e êmese, instalando um quadro de desnutrição, sendo frequentemente o primeiro sinal verificado em pacientes oncológicos, precedendo até mesmo o diagnóstico da doença (Bozzeti et al., 1982; Macedo \& Costa Rocha, 2010).

A desnutrição, promove alterações na homeostase do organismo, podendo levar a maior probabilidade de morbidade e mortalidade pós-operatória; infecção e abertura de ferida cirúrgica; sepse; dificuldade de cicatrização; aumento do tempo de internação, o que aumenta exponencialmente o custo do SUS. Cerca de 20\% das mortes por câncer são secundárias ao quadro de déficit calórico (Weimann et al., 2017).

Pacientes em tratamento quimioterápico, que apresentem quadro de desnutrição, podem apresentar pior prognóstico, levando a interrupção do tratamento; toxicidade às drogas e resposta clínica desfavorável à terapia (Dewys et al., 1980).

Diante do exposto, torna-se essencial a avaliação física regular, com aferição de peso, cálculo energético a partir das calorias por quilo de peso corporal e outros indicadores de triagem e monitoramento nutricional, específicos para pacientes oncológicos, tais como a Avaliação Subjetiva Global Produzida pelo Paciente (ASGPPP), que é capaz de detectar antecipadamente distúrbios nutricionais e pacientes com maior risco de mortalidade (Weimann et al., 2017; Gonzales et al., 2010).

Acompanhamento do Percentual de Perda de Peso Involuntária (PPI), que é um parâmetro seguro para identificação de risco nutricional, sendo considerado grave o caso em que o paciente apresentar perda ponderal de 10\% em um período de 6 meses (Gonzales et al., 2010).

Medidas antropométricas e bioquímicas, devido a fatores antinutricionais presentes conjuntamente com a doença, podem não ter a devida utilidade no rastreamento nesse perfil de pacientes Neste sentido, o acompanhamento nutricional ambulatorial deve fazer parte da rotina hospitalar, visando reverter ou manter o estado nutricional dos pacientes e os efeitos tóxicos do tratamento (Druyan et al., 2012).

\section{Terapias Integrativas}

\section{Tríade terapêutica do Câncer: Ciência, Resiliência e Fé}

A ciência, sob a ótica darwinista, trabalha com racionalidade, baseando-se em evidências, experiências, verificações e comprovações, tentando explicar o como e quando de todos os eventos naturais. Surgiu por volta do século XVI, embasada pelo Iluminismo e Renascimento, e segundo Aranha (1993), tudo aquilo que for descoberto pela ciência não pode ir de encontro aos livros sagrados.

Resiliência é a tendência que algo ou alguém tem para retornar ao seu formato/forma original, após ter sofrido modificação, qualquer que seja; habilidade de recobrar-se após ter sofrido intempéries. Para Fonagy (1994), ser resiliente é adaptar-se frente a situação estressantes, servindo de fator protetor, juntamente com outros aspectos: estilo de enfrentamento; habilidade de resolução de dificuldades; senso se eficácia, de autoconceito e de humor; autonomia e inteligência emocional; empatia; capacidade de planejamento; relacionamento parental; apoio social; boa rede de relacionamentos e experiências escolares e envolvimento em entidades religiosas.

A fé serve de inspiração para sobrepujar as dificuldades e alcançar os objetivos desejados, sendo o Espírito de Deus a força motriz que impulsiona o ser humano a continuar sonhando (Bartolomei, 2008). É fundamentada na consciência e inconsciência da crença em um ser divino, no ambiente que o cerca e no seu próprio eu. Sendo inerente ao indivíduo, compondo sua existência e psique, envolvendo emoções, vontades, desejos, sentimentos, atitudes e pessoalidade. Pereira (2016), ainda afirma que não existe fé sem razão, já que a atitude de acreditar em algo superior, esperando que está responda a seus questionamentos, inculca naquele que crê a racionalidade da ação da pura convicção.

A busca pela fé pode ter nascido da necessidade humana, devido a sua fragilidade; vazio existencial; individualismo; insegurança e incertezas; esperança e busca pelo equilíbrio, aceitando, mas não cedendo a doença, fazendo-o acreditar em sua força interior, que devido ao desconforto imposto pela doença, pode estar fragilizado (Barth, 2014; Teixeira \& Lefèvre, 2008). 
A fé não depende da lógica científica e tenta explicar o porquê e o para que da vida humana. Seu objetivo é metafisico, mas é expressada socialmente, mediante o valor perpetrado pelo indivíduo. É o campo onde a verdade absoluta manifesta-se, ou seja, onde Deus se revela, mas não precisa estar atrelada a religião (Bruscagin, 2004).

De acordo com a Igreja de Jesus Cristo dos Santos dos últimos Dias, a fé não é racional e não tira nosso livre-arbítrio, não extirpa nossas provações e desafios, mas ajuda a suportar e aprender com as adversidades, levando o indivíduo a uma autoavaliação, arrependimento e crescimento espiritual, e, para aquele que tiver a verdadeira fé, nada será impossível. E que a fé sem ação, para nada servirá, e que a ação sem a fé, menos valor terá (Rozendo, 2015).

\section{A Fé como Adjuvante Terapêutico Antitumoral}

Sob a ótica médica, a doença ataca o hospedeiro, mas, na verdade, o hospedeiro é quem está susceptível a doença e a contraí e não o contrário (Barth, 2014). Na rotina hospitalar, nem sempre é possível avaliar o paciente social, biológica, psíquica e espiritualmente, porém, esses aspectos podem ser responsáveis pelas comorbidades, aderência aos medicamentos, sucesso e fracasso no tratamento, já que dentre outras tantas doenças, está é a que causa maior abalo psicológico.

Uma saída seria, que na prática clínica fosse indicado, além de uma alimentação saudável, remédios e atividade física, a busca individual pela espiritualidade. O paciente por sua vez, é incapaz de aceitar a doença, diante da perfeição cobrada pela sociedade, onde medicamentos e aparelhos de ginástica dividem espaço (Barth, 2014). Levando a comercialização da doença e a crença de que o diagnóstico o fará passar por uma lenta e cruel marcha até a mutilação ou morte (Bartolomei, 2008). Em contrapartida, Pinto (2003), relata que mesmo com a tecnologia a favor da medicina, para alguns pacientes, a cura do câncer está atrelada ao fator milagre e não ao fator terapia.

Para Sherman (1994), os aspectos psicossociais mais relevantes são a relação médico-paciente e a visão do paciente sobre a morte. Se porventura o médico achar que o tratamento será ineficaz, mutilador, tóxico e que a morte é inexorável, a probabilidade de o paciente receber um tratamento eficaz será mínima (Bartolomei, 2008).

A força mental positiva atrelada a fé, pode aumentar as chances de um melhor prognóstico em $29 \%$ (Mieras, 2004). Visto que o estado espiritual modifica o estado físico, atuando nos sistemas: endócrino, imunológico e nervoso central (Barth, 2014).

No estudo realizado por Bartolomei (2008), todos os participantes julgaram importante o papel da fé na convivência com a doença, a equipe médica afirma, que de alguma forma, acreditar em algo é capaz de curar, dando ao indivíduo força para sobrepujar o estigma da doença e que os pacientes se sentem impelidos a acreditar em algo maior que na medicação e terapia. E um dos médicos entrevistados acrescentou que a fé não é o fator preponderante, mas que impacta positivamente no condicionamento do paciente frente ao tratamento.

Panzini (2007), explica que esse método, utilizar-se da convicção, espiritualidade e pensamentos positivos, é conhecido como coping religioso/espiritual. Mas Barth (2014), ressalta que pacientes terminais ou com doenças incuráveis não podem ser vistas como pessoas sem fé.

Está mais do que evidente, tanto na literatura cientifica quanto na não científica, os inúmeros benefícios da fé frente a aceitação, tratamento e desfecho positivo, para os pacientes com câncer. Para Costa, Oliveira e Silva (2015), aqueles que creem em algo divino, são mais saudáveis; têm pensamentos positivos, são mais otimistas e resilientes, aumentando as chances de cura. Além de serem mais felizes, terem maior sobrevida e serem mais agradáveis (Lisboa, 2013).

Wiliam Osler em 1910 escreveu que a fé derrama uma infindável corrente de vigor, fazendo com que as pessoas tenham mais vontade de viver e de buscar o melhor tratamento possível. Estudo que se harmonizou com os achados de Andrew Clark, afirmando que os adeptos a fé são mais solidários, vivem melhor socialmente, por praticarem o bem, não reagem de forma exagerada ante as adversidades e aderem melhor ao tratamento prescrito (Galilei, 2009).

Bretani, Coelho, Iyeyasu e Kowalski (1998), corrobora com esses achados, demonstrando que o diagnóstico, tratamento ou mesmo o estágio terminal do paciente podem gerar muita ansiedade e depressão, prejudicando o seu tratamento e qualidade de sobrevida, fatores que dependem diretamente de como o indivíduo encara os dilemas da vida. Assim sendo, a experiência do sofrimento pode estimular ou desestimular 
a prática religiosa. Fato encontrado por Risberg et al. (1996), onde 23\% dos entrevistados relataram uma amplificação da sua fé pós-diagnóstico. E Geronasso e Coelho (2012), que evidenciou que todos os entrevistados tiveram sua fé fortalecida mediante a instalação da doença.

Para Inzlicht, Tullet e Good (2011), os indivíduos que possuem mais fé sofrem menos de estresse, produzem maior quantidade de células imunológicas. Achados que foram corroborados por Aloustani, Mamashli e Sadeghloo (2020), que verificaram que oferecer preces antes das consultas e terapias, reduzia a pressão arterial, frequência cardíaca e respiratória dos pacientes.

Geronasso e Coelho (2012), relataram a firme convicção dos pacientes quanto a fé, como ponto central da cura da doença, até mesmo para aqueles que foram desenganados pelos médicos, verificou também, a grande adesão ao tratamento e confiança no corpo clínico, por parte dos entrevistados. Para de Serpa Junior (2003), a cura não diz respeito somente a estar livre da doença, mas, pode representar também um novo estilo de vida perpetrado pela doença, ou seja, um status quo adaptado, visto a ausência da homeostase.

Lutgendorf et al. (2004), expuseram que o risco relativo de morte diminuía 78\% e os níveis de interleucina6, 66\%, entre aqueles que visitavam instituições religiosas. De Almeida, Saad e Vieira (2008), supõem que algumas hipóteses para os inúmeros benefícios da crença sejam: reverência ao corpo; psique madura, pois suscita perdão, amor, altruísmo e esperança; aperfeiçoamento de vias psiconeuroendócrinas, psiconeuroimunológicas e psicofisiológicas, verificou também que em pacientes com câncer, o exercício da oração relacionou-se positivamente com a escala de saúde geral e de funcionalidade.

Farias e Buchalla (2005), aponta que a religiosidade pode ter implicações positivas e negativas. Positivas seriam: dieta saudável; cura para o tabagismo, alcoolismo ( $90 \%$ dos alcoolistas se afastaram das instituições religiosas ainda na mocidade) e drogas; redução de cortisol e noradrenalina através do eixo hipotálamoadrenal-hipofisário; recuperação pós-operatório mais rápida do que comparada a de ateus e agnósticos e atenuação de compulsões, paranoias, fobias e somatizações. Ponto de vista reforçado por Aquino (2014), na afirmação de que a fé pode ser positiva quando o paciente adere completamente ao tratamento. Como negativos destaca: a obstinação e algumas culturas religiosas ; práticas de jejum excessivas; automutilações e pertencer a algumas seitas religiosas que podem infringir algumas doenças mentais.

Para Fish (1973), a cura se dá pela própria ação da fé e não de um ser divino, assim sendo, a performance daqueles que pregam (autoridade religiosa que seja) é similar ao efeito de um placebo.

\section{Efeito Placebo}

Os placebos são substâncias produzidas com intuito de se parecer com os medicamentos normais, porém sem um princípio ativo, normalmente composto por amido e açúcar. Agindo mais sobre os sintomas do que na doença em si. Em latim, o termo significa "agradarei”, o termo foi achado inicialmente em um dicionário médico, em 1785, como sendo um método ou medicamento comum, posteriormente passou a ser conhecido como medicamento fictício, e atualmente, tem-se conhecido mais sobre seus efeitos ante ao enfrentamento de diversas moléstias (Stewart-Williams \& Podd, 2004).

Segundo Pereira e Farnese (2008), os remédios são usados pelos homens há muitos anos, normalmente para efeito terapêutico, complementa dizendo que o efeito placebo, mesmo não estando ligado a farmacologia em si, pode possuir implicações psicológicas para os pacientes. Rossi (1997), observa que se trata de um método de cura mente-corpo, através das crenças e esperanças a nível psicológico, podendo aliviar sintomas, como a dor, por exemplo. Para Tostes (1985), sua eficácia está mais para animar o convalescente do que tratálo e que desde sempre o efeito placebo traz efeitos positivos para a medicina, proferindo ser "maravilhosa e poderosa" a extensão da psiquê sobre a condição e as alterações da homeostase (Associados \& de Vista, 2009).

Fish (1973), relata que a prescrição de um placebo pode curar ou melhorar os sintomas através da indução psicológica, sem utilizar-se de outros medicamentos, testando o intelecto do indivíduo por meio da crença em algo ou alguém. Holden (2002), afirma que esta substância pode acionar as mesmas redes que os analgésicos, fazendo com que o fluxo sanguíneo aumente em áreas repletas de receptores de opioides, que mantém contato com diferentes regiões cerebrais, dentre eles, o córtex orbitofrontal (responsável por processar emoções). Estando envolvidos vários neurotransmissores, como a endorfina e dopamina.

Para Pereira e Farnese (2008) e Rossi (1997), sua eficácia depende da postura; do paciente e seus familiares: controle emocional, convicção em sua habilidade inata de curar-se, e sua vivência, e dos médicos: poder de convencimento, objetivo, capacitação, expectativa de cura, satisfação geral, inteligência emocional e 
prescrição terapêutica, acrescentam ainda que, a ausência de uma substância quimicamente ativa do tratamento, não afasta a hipótese de que outras variáveis e fatores sociais tenham a mesma eficácia.

Pavlov (1977), acreditava que uma explicação para o efeito placebo seria o condicionamento. Em sua experiência alguns cães foram condicionados à relacionar pessoas e sons a presença de comida, assim, a presença da pessoa ou do som, fazia com que o animal começasse a salivar, mesmo não havendo alimento no momento. Este mecanismo de resposta é similar à do organismo humano frente a presença de uma pílula de açúcar ou amido, desencadeando respostas imunológicas e hormonais.

Para Pereira e Farnese (2008), qualquer substância estranha ao organismo, ao ser ingerido, pode estimular uma resposta imunológica. Castiel (1991), acredita que cada doença está estritamente relacionada a um aspecto psicológico compatível, concebido a partir de experiência na infância, podendo surgir devido a fatores exclusivamente psíquicos, onde cada elemento possui incumbências específicas. Fato comprovado pelo surgimento da área psicossomática, que leva em consideração a somatização emocional do indivíduo, podendo desencadear sintomas físicos e moléstias (Pereira \& Farnese, 2008).

\section{Considerações finais}

Sabe-se que câncer é uma doença milenar, onipresente, metamorfoseável e extremamente adaptativa. Desponta de uma alteração e/ou ineficiência fisiológica, remodelando e readaptando tudo a sua volta, causando perturbações homeostáticas, acarretando inúmeros prejuízos para o hospedeiro.

Diante de sua multiplicidade, desde o conhecimento e entendimento da doença, procurou-se tratamentos e curas simultâneas ao surgimento de uma nova condição, descobrindo-se então terapias como a quimioterapia, radioterapia, entre outras. Inovações da medicina que tentam acompanhar as vicissitudes neoplásicas, aumentando a sobrevida dos pacientes, ainda nesse contexto, observa-se a importância de uma visão e cuidado multicêntrico e multidisciplinar, avaliando o indivíduo social, cultural, física, emocional e psicologicamente, enxergando-o como um ser altamente capacitado para participar do seu processo de tratamento e cura, respeitando suas convicções e decisões, objetivando a beneficência e a não maleficência.

E, da mesma forma que a medicina tenta acompanhar o câncer e os médicos tentam assistir aos pacientes, esses tentam lidar com o diagnóstico da doença, e assim como ela, adaptar-se a sua nova realidade, buscando meios pelos quais suas dores física, emocional e psicológica possam ser amenizadas e extirpadas, apegando-se muitas vezes a um suporte espiritual, não em prol dos tratamentos propostos, mas, muitas vezes para suportá-los e superar o sentimento de impotência e medo que o câncer causa.

A fé surge então como um adjuvante nesse processo, transcendendo o alívio espiritual e psicológico, modificando o estado físico, atuando nos sistemas: endócrino, imunológico e nervoso central, ajudando na aceitação do diagnóstico e desfechos negativos; aderência e efetividade do tratamento, até mesmo por parte dos pacientes em terminalidade. E o seu resultado, assim como o efeito placebo, se dá pelo método de cura mentecorpo, onde, através das crenças e esperanças a nível psicológico, instalam-se diversos efeitos fisiológicos, resultando em melhor qualidade de vida e sobrevida, melhor prognóstico e eficácia terapêutica.

\section{Referências}

Almeida, C. I., Saad, M., \& Vieira, M. S. R. 2008. Reabilitação em reumatologia geriátrica.

Aloustani, S., Mamashli, L., \& Sadeghloo, A. 2020. Mention the Treatment of Anger in Hospitalized Patients in the CCU department of Social Security Hospitals of Golestan Province. Indian Journal of Forensic Medicine \& Toxicology, 14(2), 1933-1937.

Aquino, T. A. A. 2014. A presença não ignorada de Deus na obra de Viktor Frankl: articulações entre logoterapia e religião. São Paulo, SP: Editora Paulus.

Aranha, M. 1993. In Arruda, Martins, M.H. Pires. Filosofando: introdução à filosofia, 2.

Associados, F., \& de Vista, P. 2009. Bases psiconeurofisiológicas do fenômeno placebo-nocebo: evidências científicas que valorizam a humanização da relação médico-paciente. Revista da Associação Médica Brasileira, 55(1), 13-8.

Barboza, C. B., \& Oliveira, A. R. L. D. 2006. Planejamento do tratamento por radioterapia através de métodos de pontos interiores. Pesquisa Operacional, 26(1), 1-24. 
Barth, W. L. 2014. A religião cura?. Teocomunicação, 44(1), 97-121.

Bartolomei, M. 2008. A fé como fator de resiliência no tratamento do câncer: uma análise do que pensam os profissionais da saúde sobre o papel da espiritualidade na recuperação dos pacientes. Trabalho de dissertação apresentado ao Programa de Estudos Pós-Graduados em Ciência da Religião. São Paulo, SP: PUC.

Bernardes, S. (2017). Prevalência e gravidade da perda ponderal em pacientes com câncer. Revista da Associação Brasileira de Nutrição-RASBRAN, 8(1), 70-74.

Bozzetti, F., Migliavacca, S., Scotti, A., Bonalumi, M. G., Scarpa, D., Baticci, F., ... \& Masserini, C. 1982. Impact of cancer, type, site, stage and treatment on the nutritional status of patients. Annals of Surgery, 196(2), 170.

Braile, D. M. 2013. A Medicina e a Fé. Brazilian Journal of Cardiovascular Surgery, 28(1), I-II.

Brentani, M. M., Coelho, F. R., Iyeyasu, H., \& Kowalski, L. P. 1998. Bases da oncologia. Bases da oncologia, 640-640.

Bruscagin, C. 2004. Família e religião. Cerveny CMO. Família, comunicação, divórcio, mudança, resiliência, deficiência, lei, bioética, doença, religião e drogadição. São Paulo, SP: Casa do Psicólogo.

Carvalho, S. C. D. 2019. Caso com nota de ensino: o acesso a imunoterapia no Sistema Único de Saúde no Brasil: um desafio complexo.

Castiel, L. D. 1991. Psicossomática e eficácia: Além do Princípio do Placebo. Jornal Brasileiro de Psiquiatria, 267-272.

Costa, J. W. N., de Oliveira Zarpelan, L., \& da Silva, J. J. A fé como propulsora para enfrentar doenças do novo século.

Coy, C. S. R. 2013. Colorectal cancer prevention in Brazil-where are we?. Journal of Coloproctology, 3(33), 111-112.

Dewys, W. D., Begg, C., Lavin, P. T., Band, P. R., Bennett, J. M., Bertino, J. R., ... Tormey, D. C. 1980. Prognostic effect of weight loss prior to chemotherapy in cancer patients. Eastern Cooperative Oncology Group. The American Journal of Medicine, 69(4), 491-497.

Druyan, M. E., Compher, C., Boullata, J. I., Braunschweig, C. L., George, D. E., Simpser, E., ... \& American Society for Parenteral and Enteral Nutrition (ASPEN) Board of Directors. 2012. Clinical guidelines for the use of parenteral and enteral nutrition in adult and pediatric patients: applying the GRADE system to development of ASPEN clinical guidelines. Journal of parenteral and enteral nutrition, 36(1), 77-80.

Farias, N., \& Buchalla, C. M. 2005. A classificação internacional de funcionalidade, incapacidade e saúde da organização mundial da saúde: conceitos, usos e perspectivas. Revista brasileira de epidemiologia, 8, 187-193.

Farris, J. R. 1996. Intervenção na crise: perspectivas teológicas e implicações práticas. Estudos de Religião, 101-118.

Fernandes, A. T. 2000. Entre a fé e a ciência: a medicina na idade média. Infecçäo hospitalar e suas interfaces na área da saúde, 43-55.

Fish, J. M. 1973. Placebo therapy: A practical guide to social influence in psychotherapy.

Fonagy, P., Steele, M., Steele, H., Higgitt, A., \& Target, M. 1994. The Emanuel Miller memorial lecture 1992 the theory and practice of resilience. Journal of Child Psychology and Psychiatry, 35(2), 231-257.

Freire, D. 2019. Imunoterapia: a virada do sistema imunológico contra o câncer. Ciência e Cultura, 71(4), 13-15.

Gabriel, G. H., Nepomuceno, L. L., de Sousa Cruz, V., \& Pimenta, E. G. D. A. 2017. Quimioterapia, Hormonioterapia e novas alternativas de tratamento do adenocarcinoma mamário. Enciclopédia Biosfera, Goiânia, 14(26), 583.

Galilei, G. 2009. Ciência e fé. São Paulo, SP: UNESP.

Geronasso, M. C. H., \& Coelho, D. 2012. A influência da religiosidade/espiritualidade na qualidade de vida das pessoas com câncer. Saúde e Meio Ambiente: revista interdisciplinar, 1(1), 173-187.

Gonzalez, M. C., Borges, L. R., Silveira, D. H., Assunção, M. C. F., \& Orlandi, S. P. 2010. Validação da versão em português da avaliação subjetiva global produzida pelo paciente. Revista Brasileira de Nutrição Clínica, 25(2), 102-8.

Hoff, P. M. G. 2013. Tratado de oncologia. São Paulo, SP: ATHENEU.

Holden, C. 2002. Drugs and placebos look alike in the brain. Science, 295(5557), 947-947.

Inzlicht, M., Tullett, A. M., \& Good, M. 2011. The need to believe: a neuroscience account of religion as a motivated process. Religion, Brain \& Behavior, 1(3), 192-212.

Júnior, A. J. S. C., de Santana, M. E., \& Sonobe, H. M. 2020.National policy for the prevention and control of cancer in the health care network of people with chronic diseases: a view based on Zygmunt Bauman 
Política nacional para la prevención y el control del cáncer en la red de atención de la. Research, Society and Development, 9(7), e413974324.

Junior, E. S., de Andrade Urban, C., de Lima, R. S., Rabinovich, I., \& Spautz, C. C. 2007. Radioterapia e quimioterapia no tratamento do câncer durante a gestação-revisão de literatura. Revista Brasileira de Cancerologia, 53(1), 41-46.

Leal, J. H. S., Cubero, D., \& Giglio, A. D. 2010. Hormonioterapia paliativa em câncer de mama: aspectos práticos e revisão da literatura. Revista da Sociedade Brasileira de Clínica Médica, 8(4), 338-343.

Lisboa, S. 2013. Fé faz bem. Revista Super Interessante, 325, 40-48.

Lutgendorf, S. K., Russell, D., Ullrich, P., Harris, T. B., \& Wallace, R. 2004. Religious participation, interleukin-6, and mortality in older adults. Health Psychology, 23(5), 465.

Macedo, A. V., \& da Costa Rocha, M. O. 2010. Avaliação e tratamento da perda de peso involuntária e significativa. Revista Médica de Minas Gerais, 20(1), 115-123.

Mieras, N. T. (2004). O relacionamento entre Fé e Medicina no tratamento do câncer Uma análise sobre a influência da fé religiosa no processo de tratamento junto a pessoas com câncer. Dissertação apresentada ao programa pós-graduação em Ciências da Religião. São Paulo, SP: Universidade Metodista de Sao Paulo.

Novaes, F. T., Cataneo, D. C., Ruiz Junior, R. L., Defaveri, J., Michelin, O. C., \& Cataneo, A. J. M. 2008. Lung cancer: histology, staging, treatment and survival. Jornal Brasileiro de Pneumologia, 34(8), 595-600.

Oliveira Santos, M. 2018. Estimativa 2018: incidência de câncer no Brasil. Revista Brasileira de Cancerologia, 64(1), 119-120.

Panzini, R. G., \& Bandeira, D. R. 2007. Coping (enfrentamento) religioso/espiritual. Archives of Clinical Psychiatry, 34, 126-135.

Pavlov, I. 1977. Classical conditioning. Learning and Instruction, 4, 26.

Pereira, D. A., \& Farnese, C. 2008. Efeito placebo, efeito nocebo e psicoterapia: correlações entre os seus fundamentos. Universitas: Ciências da Saúde, 2(1), 69-90.

Pereira, J. 2016. A fé como fenômeno psicológico (Vol. 22). Sção Paulo, SP: Escrituras Editora.

Pinto, M. H. 2003. O significado do sofrimento do paciente oncológico: narrativas dos profissionais de saúde. Tese de Doutorado. São Paulo, SP: Universidade de São Paulo.

Risberg, T., Wist, E., Kaasa, S., Lund, E., \& Norum, J. 1996. Spiritual healing among Norwegian hospitalised cancer patients and patients' religious needs and preferences of pastoral services. European Journal of Cancer, 32(2), 274-281.

Rossi, E. 1997. A psicobiologia da cura mente-corpo (AR Moraes, Trad.). Campinas: Editorial Psy II (Original publicado em 1993).

Rozendo, E. (2015). A Verdade sobre os Mórmons. São João Del-Rei, MG: Editora Baraúna.

Sawada, N. O., Dias, A. M., \& Zago, M. M. F. 2006. O efeito da radioterapia sobre a qualidade de vida dos pacientes com câncer de cabeça e pescoço. Revista Brasileira de Cancerologia, 52(4), 323-9.

Schein, C. F., Marques, A. R., Vargas, C. L., \& Kirsten, V. R. 2006. Efeitos colaterais da quimioterapia em pacientes oncológicos hospitalizados. Disciplinarum Scientia| Saúde, 7(1), 101-107.

Serpa Junior, O. D. 2003. Indivíduo, organismo e doença: a atualidade de “O Normal e o Patológico" de Georges Canguilhem.

Sherman, J. R. 1994. Aspectos psicossociais do câncer. In R. R. Love. Manual de oncologia clínica da União Internacional contra o Câncer ( $6^{\mathrm{a}}$ ed.). Nova Iorque, NY: Springer-Verlag.

Silva, P. B., Lopes, M., Trindade, L. C. T., \& Yamanouchi, C. N. 2010. Controle dos sintomas e intervenção nutricional. Fatores que interferem na qualidade de vida de pacientes oncológicos em cuidados paliativos. Revista Dor, 11(4), 282-288.

Sociedade Brasileira de Nutrição Parenteral e Enteral. (2011). Sociedade Brasileira de Clínica Médica; Associação Brasileira de Nutrologia. Projeto Diretrizes-Associação Médica Brasileira e Conselho Federal de Medicina: Terapia Nutricional na Oncologia.

Soratto, M. T., Silva, D. M., Zugno, P. I., \& Daniel, R. 2016. Espiritualidade e resiliência em pacientes oncológicos. Saúde e Pesquisa, 9(1), 53-63.

Stewart-Williams, S., \& Podd, J. 2004. The placebo effect: dissolving the expectancy versus conditioning debate. Psychological bulletin, 130(2), 324. 
Teixeira, J. J. V., \& Lefèvre, F. 2008. Significado da intervenção médica e da fé religiosa para o paciente idoso com câncer. Ciência \& Saúde Coletiva, 13, 1247-1256.

Tostes, L. R. M. 1985. O efeito placebo e os aspectos inespecíficos das psicoterapias. Jornal Brasileiro de Psiquiatria, 395-398.

Veronesi, R. 1976. Imunoterapia: o impacto médico do século. Medicina de Hoje, 2, 194.

Volgo, M. J. 1998. O sintoma entre medicina e psicanálise. O instante de dizer: o mito individual do doente sobre a medicina moderna. São Paulo, SP: Escuta.

Waitzberg, D. L., Nardi, L. D., Ravacci, G., \& Torrinhas, R. S. M. M. 2004. Síndrome da anorexia e caquexia em câncer: abordagem terapêutica. In D.L. Waitzberg. Dieta, nutrição e câncer. São Paulo, SP: Atheneu.

Weimann, A., Braga, M., Carli, F., Higashiguchi, T., Hübner, M., Klek, S., ... \& Waitzberg, D. L. 2017. ESPEN guideline: clinical nutrition in surgery. Clinical Nutrition, 36(3), 623-650.

Willis, R. A. 1952. Secondary tumours of the intestines. The Spread of Tumours in the Human Body, 210-219.

Xavier, A. T. D. F., Bittar, D. B., \& Ataíde, M. B. C. D. 2009. Crenças no autocuidado em diabetes: implicações para a prática. Texto \& Contexto-Enfermagem, 18(1), 124-130.

Xavier, B. B., \& Gentilli, R. D. M. L. 2012. Afetos e cooperação familiar como coadjuvantes do tratamento de câncer de mama em mulheres. Serviço Social em Revista, 14(2), 73-95.

\section{Minicurrículo}

Daniele Andreia Alvares. Nutricionista, formada pela Universidade Nove de Julho (2018); pós-graduada em Nutrição em Oncologia pela Faculdade Unyleya (2020); Pós-graduada em Abordagens Oncológicas na Área de Saúde pelo Centro Universitário São Camilo (2020); Pós-graduanda em Nutrição Clínica Avançada: Metabologia, Terapêutica Nutricional e Dietoterapia pela USCS (2020-2021); Mestranda Internacional em Ciências da Saúde (2020-2022). Áreas de maior interesse: Obesidade, Oncologia, Microbiota intestinal, DCNT.

Como citar: Alvares, D.A. 2020. Tratamentos antineoplásicos: foco na perspectiva holística da fé como agente terapêutico. Pubsaúde, 4, a066. DOI: https://dx.doi.org/10.31533/pubsaude4.a066

Recebido: 26 ago. 2020.

Revisado e aceito: 9 set. 2020.

Conflito de interesse: os autores declaram, em relação aos produtos e companhias descritos nesse artigo, não ter interesses associativos, comerciais, de propriedade ou financeiros que representem conflito de interesse.

Licenciamento: Este artigo é publicado na modalidade Acesso Aberto sob a licença Creative Commons Atribuição 4.0 (CC-BY 4.0). 